Relations industrielles

Industrial Relations

\title{
Loisirs et relations de travail
}

\section{Louise Dumais}

Volume 4, numéro 3, novembre 1948

URI : https://id.erudit.org/iderudit/1023445ar

DOI : https://doi.org/10.7202/1023445ar

Aller au sommaire du numéro

Éditeur(s)

Département des relations industrielles de l’Université Laval

ISSN

0034-379X (imprimé)

1703-8138 (numérique)

Découvrir la revue

Citer cet article

Dumais, L. (1948). Loisirs et relations de travail. Relations industrielles /

Industrial Relations, 4(3), 27-29. https://doi.org/10.7202/1023445ar

Tous droits réservés @ C Département des relations industrielles de l’Université Laval, 1948
Ce document est protégé par la loi sur le droit d'auteur. L'utilisation des services d'Érudit (y compris la reproduction) est assujettie à sa politique d'utilisation que vous pouvez consulter en ligne.

https://apropos.erudit.org/fr/usagers/politique-dutilisation/ 


\title{
LOISIRS ET RELATIONS DE TRAVAIL
}

\author{
Louise Dumais
}

Le problème du loisir est à l'ordre du jour. Il intéresse non seulement les hommes d'action mais aussi les penseurs, à cause de la place qu'occupe le temps libre par opposition au travail obligatoire dans la vie de l'homme moderne. Si par exemple nous considérons le travailleur, nous devons admettre que la vie de celui-ci n'est pas ce qu'elle était il y a dix ou vingt ans. Une coupure nette s'est faite entre son temps de loisir et son temps de travail.

Il n'en a pas toujours été ainsi. Les bouleversements sociaux qui ont suivi l'avènement de l'industrie ont d'abord placé le travailleur dans un état extrêmement pénible, en raison de la durée trop prolongée de son travail et des circonstances générales dans lesquelles ce travail s'accomplissait: mauvaise aération des établissements, repas trop accélérés, maniement épuisant des outils, etc. . . Ces mauvaises conditions et la trop longue durée des heures de travail entraînèrent des conséquences humaines appauvrissantes et parfois même funestes. C'est alors qu'intervinrent des associations d'ouvriers et des organismes nationaux et internationaux pour chercher à établir, dès le début du siècle présent, une législation sévère concernant le labeur quotidien.

Jadis, le travailleur peinait chaque jour douze ou quinze heures, et parfois même davantage, sans même avoir droit, dans certains pays, au repos hebdomadaire. Il peut aujourd'hui disposer de son temps après avoir travaillé durant huit ou dix heures pour gagner son pain et celui de sa famille. Certains pays ont même introduit la semaine de cinq jours et presque partout dans le monde le journalier, comme l'employé de bureau ou de magasin, bénéficie de congés payés. Une telle législation permet d'estimer qu'aux Etats-Unis, l'ouvrier contemporain jouit d'environ quatre mille heures de loisir par année. L'ouvrier canadien, pour sa part, peut disposer d'environ 3,500 heures de loisir. Ce qui est déjà très appréciable.

Et que dire maintenant si l'on envisage le problème des besoins de l'homme et le progrès sans cesse accru des techniques de production ? Certains économistes vont jusqu'à prévoir qu'une meilleure coordination du rendement des services de production en fonction de l'évaluation des besoins humains, amènerait éventuellement la réduction graduelle du travail à quelques heures par jour ou même à quelques heures par semaine. On n'a qu'à observer le chemin parcouru depuis cent ans dans le domaine du perfectionnement technique pour conclure qu'une telle perspective de travail abrégé n'a rien d'absurde.

Cette diminution constante du travail qui a suivi les excès du début de l'ère industrielle a contribué à dégager de façon plus marquée la notion « loisir» de la notion «travail». Le loisir est né du travail, il est né de la nature du travail que l'homme accomplit et des conditions dans lesquelles il exerce son métier ou sa profession.

On pourrait en donner la définition suivante: Le loisir est le temps dont l'homme peut disposer en toute liberté, après avoir satisfait par son labeur à l'obligation de gagner sa vie et celle de sa famille. C'est le temps où il lui est permis de se libérer des contraintes. Toutefois, cette liberté tend à diminuer à mesure que l'homme s'engage davantage dans des rouages sociaux qui, évidemment, exigent un certain ordre même dans le loisir. Ainsi tout individu est libre de participer ou non à certains jeux d'équipe, mais dès l'instant où il $y$ participe, il doit en observer les règles.

Pris en soi, le loisir a nettement un sens positif et quel que soit son emploi dans l'action ou dans le repos (le loisir se prend à l'état de veille du fait que l'inconscience des dormeurs empêche ceux-ci d'exercer leur libre-arbitre). Il a pour but, pour raison d'être, de contribuer à l'épanouissement de la personne humaine, c'est-à-dire, au développement et à l'équilibre harmonieux du corps et de l'esprit. Sans doute, le loisir peut se présenter comme un mouvement d'action ou comme un mouvement de détente après l'effort occasionné par le travail, mais il faudrait prendre garde de l'assimiler au désoeuvrement et à l'inertie ou encore à l'agitation, car ce sont là des états négatifs et stériles qui ne sont que la caricature du loisir. Nous devons faire des réserves aussi quant à l'organisation uniformisée ou trop poussée des services de loisirs, car ceux-ci risquent aussi de porter atteinte au développement normal de la personnalité. 
Le loisir répond à un besoin légitime de l'être humain, et comme à chaque besoin légitime correspond un droit, le loisir doit être considéré comme un objet de droit. Et en nos temps modernes, l'organisation actuelle de notre vie économique et sociale, en divisant le travail et le loisir, a confié à l'un et à l'autre un rôle comportant des obligations et des droits, et visant chacun de son côté, et relativement l'un à l'autre, à la plénitude de la vie humaine.

C'est ainsi que l'homme, tout en ayant besoin de travailler, a aussi besoin de loisir. Le péché originel a situé l'homme dans son rôle laborieux. Ployant sous le fardeau de sa chûte, l'être humain dut commencer par chercher lui-même péniblement une réponse à ses besoins primaires comme celui de manger, de se vêtir, de se chauffer. Au cours des siècles, le perfectionnement sans cesse accru de ses découvertes lui a permis, peu à peu, de se dégager de certaines servitudes tout en lui laissant le besoin de tendre vers le perfectionnement et l'équilibre de ce don de Dieu qui est la vie terrestre.

Tout homme a droit au loisir comme il a droit au travail. Il n'est pas une machine. Même s'il n'était qu'une machine on lui donnerait les soins voulus et un temps d'arrêt. Mais en tant qu'homme, il a besoin de mener une vie humaine avec tout ce que cela comporte; après avoir quitté-son travail, il a droit de bénéficier de son temps libre, suivant ses goûts et ses aptitudes, tout en se conformant aux prescriptions de la morale; il a le droit de parfaire son éducation et sa culture. 11 doit aussi avoir le temps de remplir ses obligations familiales, sociales et religieuses.

Le droit au loisir ne peut pas être considéré comme le seul privilège d'une élite ou d'une classe particulière. Car, plus le labeur quotidien aura été pénible et desséchant, plus il aura été morcelé, plus le temps réservé au loisir devra, en guise de compensation, apporter sa part d'enrichissement à la personne humaine. Le genre d'emploi du loisir devra donc être déterminé par la nature du travail effectué.

L'employé qui accomplit une tâche anonyme ne peut retirer beaucoup de fierté ni de satisfaction de son travail. A ce point de vue, le travail de l'artisan offre des possibilités beaucoup plus riches que ne le fait le travail parcellaire de certains ouvriers. L'artisan qui sculpte un meuble, la tisseuse qui, après avoir elle-même dessiné ses modèles, les réalise sur son métier, peuvent être fiers du travail de leurs mains. Ce sont là des joies que connaissent difficilement le plus grand nombre des travailleurs dans les industries mo- dernes. Cela constitue un mal dont on ne peut sous-estimer l'importance. ${ }^{1}$

Mais c'est là une des servitudes que nous a apportées le progrès. Elle existe non seulement chez les ouvriers d'usine, mais aussi chez certaines catégories de travailleurs en forêt, de petits employés de bureau et de commerce. Le premier moyen d'atténuer cette difficulté, sinon de la faire disparaître, sera sans doute celui d'intéresser les ouvriers à l'ensemble de la production, en expliquant comment l'activité de chacun s'intègre dans le plan final, et en suscitant l'esprit de collaboration. Le goût à l'ouvrage naîtra naturellement chez ceux qui auront pris conscience de leur rôle social et communautaire. Il est donc important de créer une mystique du travail pour permettre à l'ouvrier de s'épanouir.

Mais la difficulté que présente le travail moderne ne doit pas nous faire négliger l'importance du bon emploi du loisir. Car, si étonnant que paraisse ce paradoxe, il reste que, après avoir appris à travailler, l'homme doit apprendre à faire bon usage de ses temps libres. Dans ce domaine comme dans tout autre, il lui faudra acquérir des habitudes saines s'il veut être véritablement un homme libre.

Il est à remarquer que les activités de loisir ont leur répercussion sur l'individu et sur la qualité de son travail. La qualité des heures libres rend le travail lourd ou léger, triste ou joyeux.

Le loisir sainement employé aide à sublimer ses instincts, il est un rempart contre les influences pernicieuses de certains milieux, il apporte un baume aux chagrins inévitables de toute vie, enfin, il contribue à « re-créer» l'être tout entier, parce qu'il est un facteur d'équilibre après la fatigue nécessaire du travail.

Le travail, pour sa part, porte de façon si évidente la marque de l'homme, que toutes les influences agissant sur celui-ci se répercutent sur l'activité laborieuse et en déterminent la valeur.

Si à cause de la nature humaine ou d'influences du milieu et de la mauvaise qualité des loisirs offerts, ou encore de circonstances pénibles de la vie, ces heures de loisir sont déprimantes, comment l'ouvrier pourrait-il se donner à son travail d'un esprit vaillant et clairvoyant? Cela nous semble impossible, tant de patrons se plaignent de l'inattention et de l'irresponsabilité de leurs

(1) Evidemment, il ne s'agit pas de tourner le dos ̀̀ l'industrialisation et de supprimer toutes ces machines qui font la plus grande part de l'ouvrage pour ne laisser à l'homme que le rôle de manoeuvre. Mais il faut étudier de façon bien soignée la grande part des valeurs humaines que le travail moderne laisse inemployées de façon à trouver en dehors du travail un terrain propice où ces valeurs pourront s'épanouir. 
employés! Une enquête sur les modes de loisirs de ceux-ci expliquerait en grande partie leur manque d'enthousiasme sinon leur mécontentement à l'ouvrage.

Certains patrons se désintéressent complètement du comportement de leurs ouvriers dès qu'ils ont quitté lè seuil de l'usine ou du bureau. Mais si ces mêmes ouvriers, à cause d'une fatigue physiologique marquée et d'un appauvrissement intellectuel intense occasionnés par un travail desséchant accompli dans des conditions sanitaires défectueuses, ne peuvent donner à leur loisir tout le sens de récupération et d'enrichissement qu'il devrait avoir, ne seront-ils pas tentés de faire porter sur leurs employeurs la responsabilité de leur ennui, de leur fatigue ou de leur mauvaise conduite? C'est là un problème auquel nous devons porter une attention soutenue si nous ne voulons pas un jour ou l'autre avoir à faire face à des forces d'inaction ou de révolte.

Des expériences récentes survenues aux EtatsUnies ${ }^{2}$ révèlent de façon non équivoque que certains travaux, à cause de la matière première employée, ou de la position du corps pendant le travail ou encore à cause de circonstances en apparence insignifiantes, laissent des traces sur des organismes plus ou moins robustes, plus ou moins endurants, et appellent des formes particulières de loisir afin de maintenir, sinon d'améliorer, la santé des travailleurs. Certains industriels américains subissaient donc depuis quelque temps des grèves incessantes de la part de leurs employés. lls eurent l'idée de recourir aux services de psychologues et de médecins afin de découvrir la cause de ces troubles. Les recherches de ces spécialistes révélèrent que l'état de santé des ouvriers était gravement déficitaire et causait ce mécontentement général. On fit alors appel à un spécialiste en loisir qui organisa des séances régulières d'éducation physique et en fit si bien valoir les attraits et avantages que tous les travailleurs s'empressèrent d'y prendre part. Puis il organisa avec un égal succès des équipes de jeux et de sports en plein air, des excursions de fin de semaine en montagne, des camps de vacances pour les jeunes travailleurs célibataires et pour les familles, il suscita même des festivals de folklore et mit en branle un choeur de chant et un cercle de jeux dramatiques. Les employés trouvèrent à la fois une occasion d'améliorer la qualité de leur travail, de se refaire physiquement et de s'apprécier mutuellement en communiant ensemble aux sources

(2) Rapport "Industrial Recreation Conference", NewYork City (1947). de la nature, aux joies saines de la vie sportive et artistique.

Ne nous leurrons pas, nous avons un gigantesque travail d'éducation à accomplir dans ce domaine, il exigera la compréhension de tous: patrons et employés, directeurs de personnel, chefs de syndicats, mouvements paroissiaux, etc. Chez le chef d'industrie, cette compréhension devrait se manifester d'une façon indirecte afin d'éviter de donner prise aux reproches de paternalisme et d'enrégimentation que l'on entend parfois dans la bouche de certains ouvriers. Car l'employé, ne voyant souvent qu'un aspect de son bien immédiat, préférera sans doute bénéficier d'une augmentation de salaire plutôt que de se voir offrir et même imposer par les autorités des facilités de loisirs que lui-même n'aura pas demandées. Il est adulte et il entend bien employer son temps libre comme bon lui semble. C'est son droit. Il ne peut toutefois désirer ce qu'il ne connaît pas, ou ce qu'il connaît mal, mais dont il ressent un besoin inconscient. L'ouvrier a, par exemple, besoin d'une culture propre tout aussi bien que le fils du professionnel. Il souffre parfois ouvertement de constater son infériorité dans ce domaine. Et comme le temps de loisir, beaucoup plus que le temps de travail, est une occasion d'épanouissement humain, le petit employé aurait avantage à se diriger dans cette voie, et il ira d'autant plus loin qu'on l'aura aidé, éclairé et guidé.

Nous vivons à une époque de machinisme et de production accélérée qui impose des obligations et des servitudes inconnues de nos devanciers. Par contre, notre siècle, si fertile en contrastes, donne à nos employeurs le privilège de collaborer de façon efficace à l'avènement d'un véritable humanisme destiné à vaincre le prolétariat.

Mais dans le domaine des loisirs comme dans les autres, ce qui mérite d'être fait mérite d'être bien fait et les expériences concluantes réalisées aux Etats-Unis et dans d'autres pays industrialisés peuvent à l'occasion nous servir d'exemple. On a découvert que le loisir pour être facteur d'épanouissement gagne à être guidé par des travailleurs sociaux, des techniciens en loisirs dont la fonction est d'étudier en collaboration, et de réaliser avec les individus et les groupes des modes d'activités qui répondent à la nature de leurs besoins, de leurs aptitudes, de leurs goûts et leur condition sociale et matrimoniale. Par voie de conséquence ils sont un facteur de production intensive et de paix sociale.

N.D.L.R.-L'abondance de matière nous a forcé depuis plusieurs mois à retarder la publication de ce texte important. 